\title{
Escala de atitudes frente à tatuagem: elaboração e evidências de validade e precisão ${ }^{1}$
}

\author{
Scale of attitudes towards tattoos: production \\ and proof of validity and accuracy
}

\author{
Emerson Diógenes de MEDEIROS \\ Valdiney Veloso GOUVEIA ${ }^{2}$ \\ Carlos Eduardo PIMENTEL ${ }^{2}$ \\ Ana Karla Silva SOARES ${ }^{2}$ \\ Tiago Jessé Souza de LIMA²
}

\begin{abstract}
Resumo
Construiu-se uma medida de atitudes diante da tatuagem, reunindo evidências de sua validade fatorial e confiabilidade. A Escala de Atitudes frente à Tatuagem foi testada em dois estudos. No Estudo 1, participaram 273 estudantes universitários equitativamente distribuídos quanto ao gênero, com idade média de 25 anos, que responderam à Escala de Atitudes frente à Tatuagem e perguntas demográficas. Verificou-se que seus itens apresentaram poder discriminativo satisfatório, assim como emergiu a estrutura unifatorial esperada, com alfa de Cronbach de 0,96. No Estudo 2, participaram 245 estudantes universitários, a maioria do gênero masculino (55\%), com idade média de 21 anos. Todos responderam à Escala de Atitudes frente à Tatuagem e à perguntas demográficas. Por meio de análise fatorial confirmatória, corroborou-se a estrutura unifatorial indicada previamente, com alfa de Cronbach de 0,93. Conclui-se que esse instrumento reúne evidências de validade fatorial e confiabilidade, podendo ser empregado para medir atitudes diante da tatuagem entre estudantes universitários.
\end{abstract}

Unitermos: Precisão de teste. Tatuagem. Validade.

\begin{abstract}
The authors built and checked for evidence of the factorial validity and reliability of a scale for measuring attitudes towards tattoos. The Attitudes Towards Tattoos Scale was tested in two studies. In Study 1, participants comprised 273 undergraduate students, equally distributed with respect to gender, with an average age of 25. They answered the Attitudes Towards Tattoos Scale and demographic questions. It was found that the items showed adequate discriminative power, and the expected unifactorial structure was identified, with Cronbach's Alpha $(\alpha)$ of 0.96. 245 undergraduate students participated In Study 2, most of them male (55\%), with an average age of 21. They answered the Attitudes Towards Tattoos Scale and demographic questions. Based on confirmatory factor analysis, the unifactorial structure found in the previous study was corroborated, and an $\alpha$ of 0.93 was observed. These findings suggest the Attitudes Towards Tattoos Scale presents evidence of factorial validity and reliability, and may be used among undergraduate students to measure attitudes towards tattoos.
\end{abstract}

Uniterms: Test accuracy. Tattoos. Validity.

\section{MVT}

1 Apoio: Coordenação de Aperfeiçoamento de Pessoal de Nível Superior (10 autor) e demais autores bolsas, do Conselho Nacional de Desenvolvimento Científico e Tecnológico, de Produtividade em Pesquisa (processos no 302199/2008-0; 140419/2009-8; 371412/2008-0; 111766/2008-7).

2 Universidade Federal da Paraíba, Centro de Ciências Humanas, Letras e Artes, Departamento de Psicologia. Conjunto Humanístico, Bloco IV, Cidade Universitária, 58051-900, João Pessoa, PB, Brasil. Correspondência para/Correspondence to: V. V. GOUVEIA. E-mails: <vvgouveia@pequisador.cnpq.br>; <vvgouveia@gmail.com>. 
A prática da modificação corporal não é recente. De fato, é milenar o hábito de as pessoas se submeterem a transformações corporais por meio da inserção de ornamentos, colocação de piercings, escarificações e realização de tatuagens (Grumet, 1983). A literatura indica que desde tempos remotos o homem imprime pinturas e símbolos em sua pele (Nedden et al., 1994).

Diversas áreas do conhecimento têm se interessado pelo estudo da tatuagem, principalmente a antropologia (DeMello, 1993, 1995; Kent, 1997), a sociologia (Irwin, 2000; Myers, 1997; Vail, 1999), a medicina (Armstrong, DeBoer \& Cetta, 2008; Caliendo, Armstrong \& Roberts, 2004; Carrol, Rifferburgh, Roberts \& Myhne, 2002; Greif, Hewitt \& Armstrong, 1999; Mayers, Judelson, Moriarty \& Rundell, 2002; Millner \& Eichold II, 2001), a criminologia (Braithwaite, Robillard, Woodring, Stephen \& Arriola, 2001; Brooks, Woods, Knight \& Shrier, 2003) e a psicologia (Grumet, 1983; Houghton, Durkin, Parry, Turbett \& Odgers, 1996). Apesar de a temática ser discutida amplamente, no Brasil não foi encontrada qualquer referência a pesquisas empíricas sobre tatuagens ou sobre atitudes diante delas. Por exemplo, em busca realizada no Index Psi (www.bvs-psi.org.br) e Google Acadêmico (2008) com as palavras e/ou expressões-chave"tatuagem","atitudes frente à tatuagem"e "escala de atitudes frente à tatuagem", não se encontrou qualquer publicação que descrevesse pesquisa empírica que fizesse referência a um dos descritores anteriormente indicados.

Apesar de no contexto brasileiro ainda serem escassos os estudos sobre atitudes diante da tatuagem, em alguns países eles têm ocupado espaço importante das agendas de alguns pesquisadores. As atitudes diante da tatuagem vêm demonstrando ser um construto preponderante para o entendimento de diversos comportamentos e cognições, a exemplo daqueles de risco (Armstrong \& Murphy, 1997; Carrol et al., 2002), uso de substâncias, agressão (Putnins, 2002), comportamentos não saudáveis (Huxley \& Grogan, 2005), crenças religiosas (Koch, Roberts, Armstrong \& Owen, 2004a; 2004b) e promoção de saúde (Stuppy, Armstrong \& Casals-Ariet, 1998).

Em medicina, por exemplo, alguns riscos e complicações de saúde têm sido associados à modificação corporal (Armstrong, DeBoer \& Celta, 2008; Caliendo,

178 Armstrong \& Roberts, 2004; Carrol et al., 2002; Greif et al.,
1999; Mayers et al., 2002; Millner \& Eichold II, 2001), à atividade sexual pré-marital (Koch, Roberts, Armstrong \& Owen, 2005, 2007) e à infecção por HIV (Beyrer et al., 2003). Também têm sido comuns os estudos que relacionam tais atitudes com comportamentos desviantes, a exemplo do uso de drogas (Braithwaite et al., 2001; Brooks et al., 2003), e mesmo com a aparição de doenças infecciosas, como a endocardite (Armstrong, DeBoer \& Cetta, 2008; Cetta, Graham, Lichtenberg \& Warnes, 1999; Lick, Edoize, Woodside \& Conti, 2005; Shebani, Miles, Simmons \& De Giovanni, 2007; Satchithananda, Walsh \& Schofield, 2001).

As atitudes diante desse adorno corporal vêm, ainda, se mostrando adequadas para diferenciar homens e mulheres no que diz respeito a comportamentos específicos. Por exemplo, Stuppy et al. (1998) realizaram uma pesquisa na qual participaram provedores de cuidados de saúde - médicos e enfermeiros, além de estudantes de enfermagem e medicina - e verificaram que as atitudes dos participantes podem influenciar negativamente nos cuidados de saúde para com pessoas tatuadas. Esses autores observaram também que as mulheres apresentaram atitudes menos favoráveis diante de pessoas tatuadas do que o fazem seus pares do gênero oposto, fato recorrente em outros estudos (Armstrong, 1991; Hawkes, Senn \&Thorn, 2004; Schorzman, Gold, Downs \& Murray, 2006). Destaca-se, entretanto, que no contexto atual e ao menos nos Estados Unidos, o maior número de pessoas que procuram se tatuar é do sexo feminino (Armstrong, 1991; Hawkes et al., 2004; Stuppy et al., 1998).

Além dos comportamentos já citados, outros também vêm sendo associados com a tatuagem. Por exemplo, Braithwaite et al. (2001) afirmam que homens tatuados fumam mais cigarros e têm mais parceiras sexuais; já as mulheres tatuadas são mais comumente usuárias de álcool e outras drogas. Seguindo essa constatação, Deschesnes, Finès e Demers (2006) comprovam que jovens que usam tatuagem e/ou piercing incorrem num "risco natural" de apresentarem comportamentos arriscados; afirmam ainda que alguns dos fatores que contribuem para inclinação juvenil de usar tais adornos corporais são o uso de drogas, as atividades ilegais, a afiliação com gangues e os problemas com jogos de azar.

Em psicologia, parece existir uma lacuna quando se trata de estudos que consideram o assunto. Verifica- 
-se que as tatuagens estão intimamente relacionadas com comportamentos de riscos e desviantes entre jovens adolescentes e adultos, como, por exemplo, usar drogas e praticar sexo sem camisinha (Armstrong \& Murphy, 1997; Beyrer et al., 2003), sendo, portanto, importante compreendê-las. Nesse sentido, um passo preliminar seria contar com um instrumento psicometricamente adequado sobre tais atitudes, o que permitiria conhecer a força e a direção das atitudes dos jovens, favorecendo tratá-las adequadamente e definindo programas educativos que visem minorar os fatores de risco.

Encontram-se na literatura referências a um instrumento específico para medir atitudes diante do uso de tatuagem, intitulado Armstrong Tattoo Scale (ATS) (Armstrong, Owen, Roberts \& Koch, 2002; Stuppy et al., 1998). Inicialmente, ele era composto por 17 itens respondidos de acordo com uma escala de tipo diferencial semântico, tendo apresentado parâmetros psicométricos satisfatórios. Por exemplo, em cinco amostras estadunidenses independentes, coerentemente, a escala apresentou uma estrutura unifatorial e consistência interna (alfa de Cronbach) variando de 0,92 a 0,95 (Stuppy et al., 1998). Porém, seus itens não foram encontrados na literatura consultada. Existe uma versão modificada da ATS, composta por 30 itens (Greif et al., 1999), que faz parte do Armstrong Tattoo Attitude Survey (ATTAS) (Armstrong et al., 2002). Apesar disso, não se encontrou qualquer referência acerca de sua adequação psicométrica.

Em resumo, considerando a importância das atitudes diante da tatuagem, sobretudo em razão de elas serem boas preditoras de comportamentos (Ajzen, 2001; Ajzen \& Fishbein, 2005), justifica-se pensar na elaboração de um instrumento específico para medi-las, conhecendo evidências empíricas acerca de seus parâmetros psicométricos. Como previamente foi indicado, quer na literatura internacional quer na brasileira, não se encontrou qualquer medida específica cujas informações técnicas estejam disponíveis. Os estudos a seguir descritos têm como objetivos principais elaborar e reunir evidências de validade fatorial e consistência interna de uma medida nova sobre atitudes diante da tatuagem. Tais estudos foram aprovados pelo Comitê de Ética em Pesquisa com Seres Humanos, do Hospital Universitário Lauro Wanderley (CEP/HULW), do Centro de Ciências da Saúde, da Universidade Federal da Paraíba, protocolo n 0011, em 28 de maio de 2008, estando de acordo com a Resolução No 196/96, do Conselho Nacional de Saúde/Ministério da Saúde - CNS/MS.

\section{Método}

\section{Estudo 1. Elaboração da escala de atitudes frente à tatuagem}

Esse primeiro estudo teve como objetivo principal elaborar a Escala de Atitudes frente à Tatuagem (EAFT-D). Procurou-se elaborar os itens, avaliar o poder discriminativo de cada um deles e conhecer a estrutura fatorial e a consistência interna do instrumento.

\section{Participantes}

Contou-se com uma amostra não probabilística - intencional, de conveniência -, formada por 273 estudantes de uma universidade particular da cidade de João Pessoa (PB), com idade Média (M) de 25 anos e Desvio-Padrão - DP (DP) de 6,73 (amplitude de 17 a 50 anos), sendo quase igualmente distribuídos quanto ao gênero (masculino=51,7\%; feminino=49,3\%).

\section{Instrumentos}

Os participantes responderam a um questionário com duas partes principais:

A Escala de Atitudes frente à Tatuagem (EAFT-D) compõe-se de 10 itens, representando pares de adjetivos que expressam avaliações gerais diante da tatuagem. Alguns deles foram retirados de Crites, Fabrigar e Petry (1994) - por exemplo, agradável/desagradável, desejável/ indesejável e positivo/negativo - e se mostraram eficientes para medir atitudes com relação a vários objetos, tendo sido empregados previamente no contexto brasileiro para avaliar atitudes diante da maconha (Gouveia, Pimentel, Queiroga, Meira \& Jesus, 2005) e das drogas em geral (Gouveia, Pimentel, Medeiros, Gouveia \& Palmeira, 2007). Os itens restantes foram elaborados pelos autores. Como parece evidente, a EAFT-D se baseia em diferencial semântico, sendo as respostas expressas em escala de 5 pontos (+2 a -2, tendo o "zero" como 
ponto médio da escala). Além dos três itens previamente listados, incluíram-se os sete seguintes: certo/errado; adequado/inadequado; responsável/irresponsável, delicado/agressivo, pacífico/rebelde, bonito/feio e convencional/anticonvencional. Antes de responder aos itens, os participantes têm em conta a seguinte frase: "Considero estar usando tatuagens..."

Todos os participantes responderam a perguntas que procuravam caracterizá-los quanto ao curso, ao sexo e à idade.

Elaborada a versão preliminar da EAFT-D, realizou-se primeiramente sua validação semântica, levada a cabo com a participação de 20 estudantes do primeiro período do curso de administração. Nessa oportunidade, procurou-se verificar se as instruções sobre como responder, se o formato da escala de resposta e se os itens em si eram compreensíveis. Verificado que não ocorreram questionamentos, manteve-se a versão proposta.

\section{Procedimentos}

Com o fim de realizar a coleta de dados, contatou-se inicialmente o coordenador pedagógico da universidade particular, escolhida por conveniência, procurando obter a permissão para aplicação dos questionários. Após o consentimento da coordenação, a aplicação foi efetuada por três bolsistas de Iniciação Científica (IC) do curso de psicologia, previamente treinados, que foram instruídos a se limitarem às instruções da escala e a esclarecerem os respondentes quanto à forma, mas não ao conteúdo da medida.

Os instrumentos, de natureza autoaplicável, foram aplicados de forma coletiva em sala de aula, porém respondidos individualmente. Os participantes foram informados acerca do caráter voluntário de sua participação e que seriam garantidos o anonimato e o sigilo das respostas. Esclareceram-se as diretrizes éticas que regem as pesquisas com seres humanos, oportunidade em que os participantes foram solicitados a assinar um termo de consentimento livre e esclarecido e rubricar o questionário antes de devolvê-lo preenchido. Em média, 20 minutos foram suficientes para completar a participação.

O Statistical Package for the Social Sciences (SPSS) (versão 15) foi utilizado para analisar os dados e esta- tísticas descritivas (medidas de tendência central e dispersão, distribuição de frequência) para caracterizar os participantes do estudo. Com o fim de conhecer o poder discriminativo dos itens, empregou-se o teste $t$ de Student para grupos independentes. A análise fatorial exploratória, por meio do método dos eixos principais (Principal Axis Factoring), foi empregada para conhecer a estrutura fatorial da escala. O alfa de Cronbach foi o índice de consistência interna adotado, permitindo verificar a coerência que cada item tem com os demais que compõem o instrumento.

\section{Resultados 1}

Os resultados são apresentados em subseções, organizadas segundo as análises efetuadas. Primeiramente é apresentado o poder discriminativo de cada item e, logo em seguida, a análise fatorial exploratória e consistência interna da escala.

\section{Poder discriminativo dos itens}

Nessa oportunidade, partiu-se do critério de mediana empírica para definir os grupos-critério. Portanto, somando-se todos os itens, foi possível obter a pontuação total da escala. Com base nela, definiram-se os grupos inferior e superior, conforme as pontuações totais dos participantes fossem abaixo ou acima da mediana respectivamente. Para cada item, foi calculado um teste $t$ comparando as médias dos dois grupos (Tabela 1).

Os resultados dos testes $t$ indicam que todos os itens apresentaram poder discriminativo satisfatório $(p<0,001)$. Portanto, lograram diferenciar os participantes dos dois grupos-critério (inferior e superior) com magnitude e direção esperadas. Esse aspecto assegura a qualidade métrica dos itens desse instrumento, podendo, assim, diferenciar pessoas com pontuações próximas. Partiu-se, então, para a verificação de evidências de sua validade fatorial e consistência interna.

\section{Análise fatorial exploratória}

Procurou-se previamente conhecer a fatorabilidade da matriz de correlações entre os itens da escala, empregando-se o Kaiser-Meyer-Olkin (KMO) e o Teste de Esfericidade de Bartlett. $O$ primeiro trabalha com as 
Tabela 1. Poder discriminativo dos itens da EAFT-D.

\begin{tabular}{|c|c|c|c|c|c|c|}
\hline \multirow{3}{*}{ Itens } & \multicolumn{4}{|c|}{ Grupo-critério } & \multirow{2}{*}{\multicolumn{2}{|c|}{ Contraste }} \\
\hline & \multicolumn{2}{|c|}{ Inferior } & \multicolumn{2}{|c|}{ Superior } & & \\
\hline & M & DP & $M$ & $\mathrm{DP}$ & $t(269)$ & $p$ \\
\hline 1. Positivo/Negativo & $-0,91$ & 0,99 & 0,87 & 0,99 & $-14,90$ & 0,001 \\
\hline 2. Agradável/Desagradável & $-0,69$ & 0,98 & 1,06 & 0,81 & $-15,64$ & 0,001 \\
\hline 3. Desejável/Indesejável & $-0,78$ & 1,11 & 1,09 & 0,88 & $-14,90$ & 0,001 \\
\hline 4. Bonito/Feio & $-0,44$ & 1,21 & 1,41 & 0,71 & $-14,59$ & 0,001 \\
\hline 5. Delicado/Agressivo & $-0,96$ & 0,88 & 0,68 & 0,98 & $-14,35$ & 0,001 \\
\hline 6. Certo/Errado & $-0,97$ & 0,95 & 0,71 & 0,92 & $-14,48$ & 0,001 \\
\hline 7. Responsável/Irresponsável & $-0,80$ & 0,96 & 0,69 & 1,02 & $-12,31$ & 0,001 \\
\hline 8. Adequado/Inadequado & $-1,14$ & 0,87 & 0,50 & 0,99 & $-14,47$ & 0,001 \\
\hline 9. Pacífico/Rebelde & $-0,94$ & 0,95 & 0,53 & 1,00 & $-12,27$ & 0,001 \\
\hline 10. Convencional/Anticonvencional & $-1,05$ & 0,92 & 0,41 & 1,13 & $-11,67$ & 0,001 \\
\hline
\end{tabular}

M: média; DP: desvio-padrão; EAFT-D: escala de atitudes frenta à tatuagem.

correlações parciais das variáveis, devendo ser aceito valor de KMO igual ou superior a 0,60 (Tabachnick \& Fidell, 2006); o segundo comprova a hipótese de que a matriz de covariâncias é de identidade, isto é, apresenta 1 (uns) na diagonal e 0 (zeros) no restante da matriz. Valores significativos indicam que essa hipótese é rejeitada, sendo adequado realizar uma análise fatorial. Os resultados apoiaram esse tipo de análise estatística: $\mathrm{KMO}=0,95$ e Teste de Esfericidade de Bartlett, $\chi^{2}$ $(45)=2.2356,56 ; p<0,001$. Nesse sentido, decidiu-se adotar o método PAF (Principal Axis Factoring), sem fixar tipo de rotação ou número de fatores a extrair. Isso permitiu identificar um único fator com valor próprio (eigenvalue) superior a 1 (Critério de Kaiser), explicando 69,5\% da variância total.

Somente um valor próprio é destacadamente discrepante dos restantes, como fica evidenciado pela forma de cotovelo que se configura a partir do segundo valor próprio (Figura 1). Portanto, ao traçar uma linha (pontilhada), é possível observar que os demais valores próprios quase não se diferenciam uns dos outros, confirmando uma estrutura unifatorial. Não obstante, com o fim de dirimir qualquer dúvida, realizou-se uma Análise Paralela (Hayton, Allen \& Scarpello, 2004). Nesse caso, assumiram-se as mesmas características do banco de dados empíricos: 273 participantes e 10 itens, realizando mil simulações. Os valores próprios gerados aleatoriamente foram comparados com os obtidos na análise fatorial, confirmando a presença de um único fator. Um único valor próprio observado foi superior ao obtido com a análise paralela. Os resultados da PAF são mostrados na Tabela 2.
Tabela 2. Estrutura fatorial da escala de atitudes frente à tatuagem (EAFT-D).

\begin{tabular}{|c|c|c|}
\hline Itens & Conteúdo de itens & Carga \\
\hline 06 & Certo/Errado & 0,89 \\
\hline 02 & Agradável/Desagradável & 0,88 \\
\hline 08 & Adequado/Inadequado & 0,88 \\
\hline 07 & Responsável/irresponsável & 0,84 \\
\hline 05 & Delicado/Agressivo & 0,84 \\
\hline 09 & Pacífico/Rebelde & 0,83 \\
\hline 03 & Desejável/Indesejável & 0,82 \\
\hline 04 & Bonito/Feio & 0,81 \\
\hline 01 & Positivo/Negativo & 0,78 \\
\hline \multirow[t]{5}{*}{10} & Convencional/Anticonvencional & 0,74 \\
\hline & & 10 \\
\hline & & 7,24 \\
\hline & & 69,5 \\
\hline & & 0,96 \\
\hline
\end{tabular}

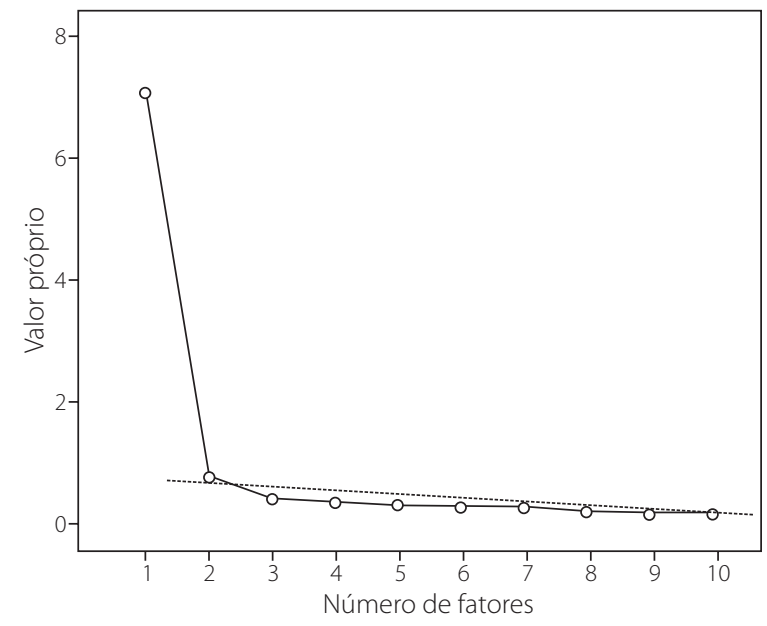

Figura 1. Representação gráfica dos valores próprios. 
O fator geral de atitudes diante da tatuagem teve valor próprio de 7,24, com seus itens apresentando saturações variando de 0,74 (Item 10. Convencional/ Anticovencional) a 0,89 (Item 06. Certo/Errado). Portanto, pode-se assumir que os itens desse fator geral refletem atitudes diante da tatuagem, recebendo essa denominação. Seu índice de consistência interna (alfa de Cronbach) foi de 0,96.

\section{Discussão parcial}

Este estudo procurou elaborar a medida de atitudes diante da tatuagem. Seus itens funcionaram adequadamente, conseguindo diferenciar os participantes com baixa e alta pontuação na escala, mesmo considerando um critério mais exigente como o da mediana (Pasquali, 2003). Evidenciou-se tratar de uma estrutura unifatorial, confirmada por meio de diversos critérios, inclusive da análise paralela, considerada mais robusta (Haylton et al., 2004). As saturações dos itens foram acima de |0,30|, valor que tem sido recomendo na literatura (Gorsuch, 1983; Pasquali, 2003). Além disso, a consistência interna dessa medida se mostrou satisfatória, superior também ao ponto de corte comumente sugerido de 0,70 (Nunnally, 1991).

Apesar de esses resultados indicarem evidências empíricas de validade fatorial e consistência interna da EAFT-D, há de se frisar que as análises realizadas foram estritamente exploratórias. Nenhuma avaliação foi feita acerca do modelo unifatorial derivado empiricamente. Esse aspecto motivou um segundo estudo, tratado a seguir.

\section{Estudo 2. confirmando a validade fatorial e consistência interna da EAFT-D}

Como anteriormente sugerido, este estudo procura ir além do anterior, contribuindo com procedimentos confirmatórios para checar a adequação da EAFT-D. Concretamente, objetivou testar a estrutura fatorial dessa medida e verificar a estabilidade do seu alfa de Cronbach.

\section{Participantes}

Participaram 245 estudantes de diversos cursos 182 de uma instituição de ensino superior pública da cidade de João Pessoa (PB), com idade média de 21 anos (DP=3,58; amplitude de 18 a 54), sendo a maioria do gênero masculino $(55,0 \%)$, solteira $(93,0 \%)$ e se declarando de classe média (60,8\%).

\section{Instrumentos}

Os participantes responderam a um questionário com duas partes: Escala de Atitudes frente à Tatuagem (EAFT-D), detalhadamente descrita no Estudo 1, e perguntas demográficas quanto ao sexo, ao estado civil eà idade.

\section{Procedimentos}

Inicialmente, entrou-se em contato com coordenadores dos cursos para solicitar a permissão para a coleta de dados. Com o consentimento obtido, visitaram-se as salas de aula, escolhidas por conveniência, onde foi realizada a coleta. A aplicação foi efetuada por dois bolsistas de iniciação científica do curso de psicologia, previamente treinados. Embora as resposta tenham sido individuais, a coleta foi realizada em ambiente coletivo de sala de aula. Todos foram informados acerca do anonimato e sigilo da participação, sendo solicitados a rubricar um termo de consentimento livre e esclarecido. Em média, 20 minutos foram suficientes para concluir a atividade.

Para comprovação da estrutura fatorial da EAFT-D, o AMOS (versão 7) foi utilizado. No caso, por meio de modelagem por equações estruturais, testou-se a estrutura fatorial previamente observada (Estudo 1), tomando como referência alguns indicadores de ajuste do modelo teórico com relação aos dados empíricos (Byrne, 2001; Tabachnick \& Fidell, 2006): 1) o qui-quadrado, que testa a probabilidade de o modelo teórico se ajustar aos dados; quanto maior esse valor, pior o ajustamento. Por ser sensível ao tamanho da amostra, deve-se interpretá-lo com reserva, valendo-se de sua razão em relação aos graus de liberdade ( $\chi^{2} / g$.l.). Nesse caso, valores entre 2 e 3 indicam um ajustamento adequado, sendo aceito um valor de até 5 ; 2) o Goodnessof-Fit Index (GFI) e o Adjusted Goodness-of-Fit Index (AGFI), que variam de 0 a 1, com valores na casa dos 0,90 ou superiores indicando um ajustamento satisfatório; 3) o Comparative Fit Index (CFI), que é um índice comparativo, 
adicional, de ajuste ao modelo, com valores mais próximos de 1 indicando melhor ajuste; recomendam-se valores de 0,90 ou superiores; e 4) a Root-Mean-Square Error of Approximation (RMSEA), com seu intervalo de confiança de 90\% (IC90\%); seu valor deve ser igual ou inferior a 0,08, aceitando-se valores de até 0,10. Para comparar os alfas de Cronbach, teve-se em conta o teste de diferença $(\Delta F)$ para alfas de amostras independentes (van der Vijver \& Leung, 1997).

\section{Resultados 2}

Com o fim de realizar a análise fatorial confirmatória, teve-se em conta a matriz de covariância, adotando-se o estimador ML (Máxima Verossimilhança). Seguindo o observado no estudo anterior, definiram-se todos os itens como saturando em um único fator. Os indicadores de qualidade de ajuste, apesar de não terem sido extraordinários, foram promissores: $\chi^{2}(35)=160,46$, $p<0,001 ; \chi^{2} / g l=4,58, \mathrm{GFI}=0,86, \mathrm{AGFI}=0,78, \mathrm{CFI}=0,92 \mathrm{e}$ RMSEA=0,12 (IC90\%=0,10-0,14). Não obstante, decidiu-se reespecificar o modelo, considerando os IMs (Índices de Modificação). Concretamente, reespecificaram-se as covariâncias entre dois pares de erros de medida: itens 1 (Certo/Errado) e 4 (Responsável/Irresponsável), por um lado, e 7 (Desejável/Indesejável) e 8 (Bonito/Feio), por outro. Com essas modificações, obtiveram-se melhores índices de ajuste: $\chi^{2}(33)=84,62, p<0,001 ; \chi^{2} / g l=2,56$, $\mathrm{GFI}=0,93, \mathrm{AGFI}=0,88, \mathrm{CFI}=0,97$ e $\mathrm{RMSEA}=0,08$ (IC90\%=0,06-0,10). A estrutura fatorial correspondente pode ser vista na Figura 2.

Os pesos fatoriais (Lambdas) são todos positivos e estatisticamente diferentes de zero $(\lambda>0,50$; $t>3,92$, $p<0,001$ ) (Figura 2). Portanto, os índices de bondade de ajuste admitem apoio para a estrutura unidimensional da medida de atitude diante da tatuagem. Resta, não obstante, checar a consistência interna dessa estrutura. Nesse caso, não somente foi calculado o alfa de Cronbach, mas também ele foi comparado com o observado no Estudo 1, checando a possibilidade de corroborá-lo. Nessa oportunidade, constatou-se um alfa de Cronbach de 0,93. Comparando esse coeficiente com o encontrado no estudo anterior $(0,96)$, constata-se uma diferença estatisticamente significativa $[\Delta \mathrm{F}(244$, 272) $=1,75, p<0,001]$.

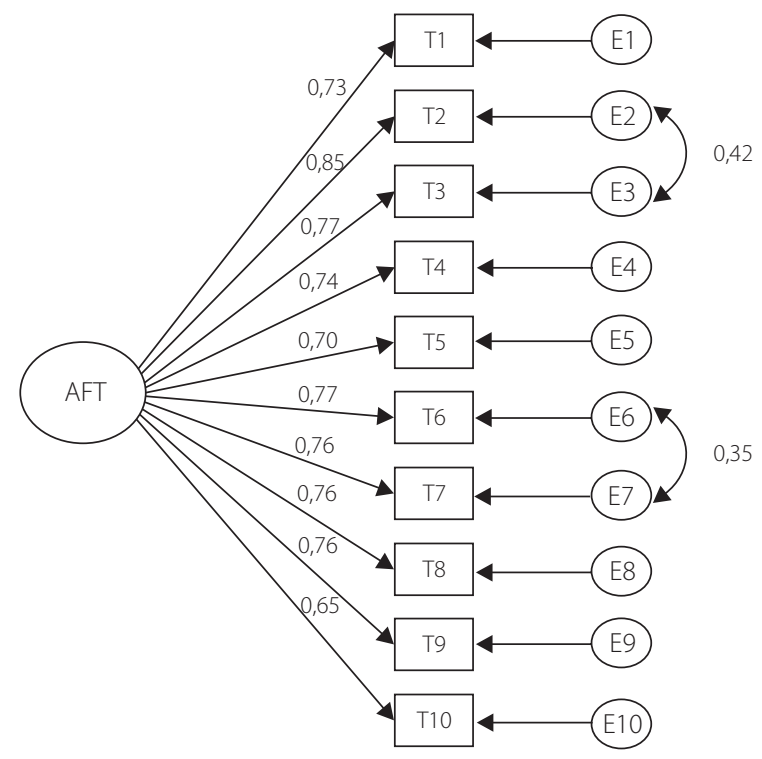

Figura 2. Estrutura Fatorial da Escala de Atitudes Frente à Tatuagem.

\section{Discussão parcial}

Este estudo reúne provas complementares e robustas, partindo de análises estatísticas confirmatórias sobre a estrutura fatorial e consistência interna da medida de atitudes diante da tatuagem. Portanto, claramente a EAFT-D se revelou unifatorial, sendo composta por itens que acentuam uma dimensão homogênea, refletida no índice de consistência interna observado.

\section{Discussão}

O objetivo principal deste artigo foi apresentar uma medida nova de atitudes diante da tatuagem, reunindo evidências de sua validade fatorial e consistência interna. Confia-se que tenha sido alcançado. Os dois estudos realizados utilizam um instrumento que necessita apenas de lápis e papel, sendo simples e autoaplicável, cujos itens isoladamente e no conjunto apresentam qualidades métricas satisfatórias, possibilitando seu emprego em pesquisas futuras.

Apesar do anteriormente comentado, reconhece-se, como em qualquer empreendimento científico, que os estudos levados a cabo não estão isentos de limitações potenciais. Consideraram-se, por exemplo, apenas estudantes universitários, constituindo-se amostras muito específicas e de conveniência. Desse modo, 
reconhece-se que os resultados descritos não podem ser extrapolados para outros grupos amostrais. Contudo, não se pretendeu generalizar os resultados, mas sim conhecer se a medida proposta apresentava parâmetros psicométricos adequados.

A estrutura fatorial encontrada está dentro do que seria recomendado na literatura fundamentada na teoria clássica dos testes (Pasquali, 1999, 2003). Essa escala explicou mais de 2/3 da variância total das respostas, apresentando índice de consistência interna superior a 0,70, valor preconizado na literatura (Nunnally, 1991; Pasquali, 2003). Seus parâmetros psicométricos são similares aos apresentados para a versão original da ATS (Stuppy et al., 1998), que conta com quase o dobro de itens, igualmente avaliados em escala de diferencial semântico. Coerentemente, ambos os instrumentos se mostraram unidimensionais, com índices de consistência interna superiores a 0,90 (alfas de Cronbach). 0 fato de no Estudo 2 o alfa ser significativamente inferior àquele do Estudo 1 deve merecer atenção. Talvez diferenças demográficas ou mesmo culturais possam explicar a variação desses coeficientes. Entretanto, ainda que o do último estudo tenha sido inferior, foi claramente bem acima dos valores geralmente encontrados para escalas de atitudes. Não é possível uma comparação da EAFT-D com a versão vigente da ATS (30 itens), pois não se encontrou qualquer publicação que reportasse seus parâmetros.

Replicar a presente pesquisa, considerando outros contextos, poderia ser um passo importante no futuro. Seria interessante, por exemplo, contar com a participação de jovens com e sem tatuagens, checando se a EAFT-D é capaz de diferenciar tais grupos. Nesse mesmo contexto, caberia checar que pontuação dos participantes permitiria maximizar a sensibilidade e especificidade dessa medida, qualidades métricas que têm sido pouco levadas em conta na literatura psicológica. Poder-se-ia também testar a invariância fatorial dessa medida nesses dois grupos, ou tendo em conta o sexo dos participantes, variável em função da qual os jovens se diferenciam nas atitudes diante da tatuagem (Hawkes et al., 2004; Schorzman et al., 2006). Caberia ainda reunir evidências de validade preditiva dessa medida, corroborando o que preconiza a literatura sobre serem

184 as atitudes boas preditoras da intenção ou do compor- tamento propriamente dito (Ajzen, 2001; Ajzen \& Fishbein, 2005).

Finalmente, a EAFT-D poderá ser adequadamente empregada em pesquisas futuras com amostras semeIhantes às aqui consideradas. A propósito, sugere-se seu uso no âmbito educacional atrelado a medidas que enfoquem aspectos ou conteúdos quotidianos de adolescentes e jovens adultos, como preferência musical (Pimentel, 2004), condutas antissociais e delitivas (Santos, 2008) ou atitudes diante do uso de drogas e/ou álcool (Gouveia et al., 2005; Gouveia et al., 2007). Isso contribuiria para dimensionar as implicações de uma atitude favorável ou desfavorável diante da tatuagem. Além disso, não se descarta empregá-la como medida de screening (triagem), que, conjuntamente com outras, permitiria identificar grupos potencialmente de risco, quer no âmbito educacional (Fonseca, 2008), quer no da saúde (Armstrong \& Murphy, 1997; Armstrong, Roberts, Owen \& Koch, 2004; Brooks et al., 2003).

\section{Referências}

Ajzen, I. (2001). Nature and operation of attitudes. Annual Review Psychology, 52 (1), 27-58.

Ajzen, I., \& Fishbein, M. (2005). The influence of attitudes on behavior. In D. Albarracín, B. T. Johnson \& M. P. Zanna (Orgs.), The handbook of attitudes (pp.173-221). New Jersey: Lawrence Erlbaum.

Armstrong, M. L. (1991). Career-oriented women with tattoos. Journal of Nursing Scholarship, 23 (4), 215-220.

Armstrong, M. L., DeBoer, R. N., \& Cetta, F. (2008). Infective endocarditis after body art: a review of the literature and concerns. Journal of Adolescent Health, 43 (3), 217-225.

Armstrong, M. L., \& Murphy, K. P. (1997). Tattooing: another adolescent risk behavior warranting health education. Applied Nursing Research, 10 (4), 181-189.

Armstrong, M. L., Owen, D. C., Roberts, A. E., \& Koch, J. R. (2002). College students and tattoos: influence of image, identity, family, and friends. Journal of Psychosocial Nursing, 40 (10), 21-29.

Armstrong, M. L., Roberts, A. E., Owen D. C., \& Koch, J. R. (2004). Toward building a composite of college student influences with body art. Issues in Comprehensive Pediatric Nursing, 27 (4), 277-295.

Attitudes Toward Tattoo. (2008). Retrieved June 6, 2008, from http://scholar.google.com.br/_schhp?sourceid= navclient\&hl=pt-BR

Beyrer, C., Jittiwutikarn, J., Teokul, W., Razak, M. H., Suriyanon, V., Srirak, N., et al. (2003). Drug use, increasing 
incarceration rates, and prison-associated HIV risk in Thailand. AIDS and Behavior, 7 (2), 153-161.

Braithwaite, R., Robillard, A., Woodring, T., Stephen, T., \& Arriola, K. J. (2001). Tattooing and body piercing among detainees: relationship to alcohol and other drug use. Journal of Substance Abuse, 13 (1-2), 5-16.

Brooks, T. L., Woods, E. R., Knight, J. R., \& Shrier, L. A. (2003). Body modification and substance use in adolescents: is there a link? Journal of Adolescent Health, 32 (1), 44-49.

Byrne, B. M. (2001). Structural equation modeling with amos: basic concepts, applications, and programmimg. New York: Springer-Verlag.

Caliendo, C., Armstrong, M. L., \& Roberts, A. E. (2004). Selfreported characteristic of women and men with intimate body piercings. Journal of Advanced Nursing, 49 (5), 474-484

Carrol, S. T., Riffenburgh, R. H., Roberts, T. A., \& Myhne, E. B. (2002). Tattoos and body piercings as indicator of adolescent risk-taking behavior. Pediatrics, 109 (6), 1021-1027.

Cetta, F., Graham, L. C., Lichtenberg, R. C., \& Warnes, C. A. (1999). Piercing and tattooing in patients with congenital heart disease: patient and physician perspectives. Adolescent Health Brief, 24 (3), 160-162.

Crites S. L., Fabrigar L. R., \& Petty R. E. (1994). Measuring the affective and cognitive properties of attitudes: conceptual and methodological issues. Personality and Social Psychology Bulletin, 20 (6), 619-634.

DeMello, M. (1993). The convict body: tattooing among male American prisoners. Anthropology Today, 9 (6), 10-13.

DeMello, M. (1995). Not just for bikers anymore: popular representations of American tattooing. Journal of Popula Culture, 29 (3), 37-52

Deschesnes, M., Finès, P., \& Demers, S. (2006). Are tattooing and body piercing indicators of risk-taking behaviours among high school students? Journal of Adolescence, 29 (3) 379-393.

Fonseca, P. N. (2008). Desempenho acadêmico de adolescentes: proposta de um modelo explicativo. Tese de doutorado não-publicada, Universidade Federal da Paraíba, João Pessoa.

Gorsuch, R. (1983). Factor analysis. Hillsdale, N.J.: Lawrence Erlbaum Associates.

Gouveia, V. V., Pimentel, C. E., Medeiros, E. D., Gouveia, R. S. V., \& Palmeira, J. (2007). Escala de atitudes frente ao uso de drogas: evidências de validade fatorial e preditiva. Jornal Brasileiro de Psiquiatria, 56 (1), 53-59.

Gouveia, V. V., Pimentel, C. E., Queiroga, F., Meira, M., \& Jesus, G. R. (2005). Escala de atitudes frente ao uso de maconha: comprovação de sua validade de construto. Jornal Brasileiro de Psiquiatria, 54 (1), 5-12.

Greif, J., Hewitt, W., \& Armstrong, M. L. (1999). Tattooing and body piercing: body art practices among college students. Clinical Nursing Research, 8 (4), 368-385.

Grumet, G. W. (1983). Psychodynamic implications of tattoo. American Journal of Orthopsychiatry, 53 (3), 482-492.
Hayton, J. C., Allen, D. G., \& Scarpello, V. (2004). Factor retention decisions in exploratory factor analysis: a tutorial on parallel analysis. Organizational Research Methods, 7 (2), 191-205.

Hawkes, D., Senn, C. Y., \& Thorn, C. (2004). Factors that influence attitudes toward women with tattoos. Sex Roles, 50 (9/10), 593-604.

Houghton, S., Durkin, K., Parry, E., Turbett, Y., \& Odgers, P. (1996). Amateur tattooing practices and beliefs among high school adolescents. Journal of Adolescent Health, 19 (6), 20-425.

Huxley, C., \& Grogan, S. (2005). Tattooing, piercing, healthy behaviours and health value. Journal Health Psychology, 10 (6), 831-841.

Irwin, K. (2000). Negotiating the tattoo. In P. Adler \& P. Adler (Eds.), Constructions of deviance (pp.459-470). Belmont, CA: Wadsworth.

Kent, D. (1997). Decorative bodies: the significance of convicts' tattoos. Journal of Australian Studies, 53 (1), 78-88.

Koch, J. R., Roberts, A. E., Armstrong, M. L., \& Owen, D. C. (2004a). Correlations of religious belief and practice with college student's tattoo-related behavior. Psychological Reports, 94 (2), 425-430.

Koch, J. R., Roberts, A. E., Armstrong, M. L., \& Owen, D. C. (2004b). Religious belief and practice in attitudes toward individuals with body piercing. Psychological Reports, 95 (2), 583-586.

Koch, J. R., Roberts, A. E., Armstrong, M. L., \& Owen, D. C. (2005). College students, tattoos, and sexual activity, Psychological Reports, 97 (3), 887-890.

Koch, J. R., Roberts, A. E., Armstrong, M. L., \& Owen, D. C. (2007). Frequencies and relations of body piercing and sexual experience in college students. Psychological Reports, 101 (1), 159-162.

Lick, S. D., Edoize, S. N., Woodside, K. J., \& Conti, V. R. (2005). Streptococcus viridans endocarditis form tongue piercing. Journal of Emergency Medicine, 29 (1), 57-59.

Mayers, L. B., Judelson, D. A., Moriarty, B. W., \& Rundell, K. W. (2002). Prevalence of body art (body piercing and tattooing) in university undergraduates and incidence of medical complications. Mayo Clinic Proceedings, 77 (1), 29-34.

Millner, V. H., \& Eichold II, B. H. (2001). Body piercing e tattooing perspective. Clinical Nursing Research, 10 (4), 424-441.

Myers, J. (1997). Nonmainstream body modification. In P. Adler \& P. Adler (Eds.), Constructions of deviance (pp.516-532). New York: Wadsworth.

Nedden D. N., Wicke, K., Knapp, R., Seidler, K., Wilfing, H., Weber, G., et al. (1994). New findings on the Tyrolean "Ice Man": archaeological and CT-Body analysis suggest personal disaster before death. Journal of Archaeological Science, 21 (6), 809-818.

Nunnally, J. C. (1991). Teoría psicométrica. México, DF: Trilhas.

Pasquali, L. (1999). Instrumentos psicométricos:manual prático de elaboração. Brasília: LabPAM/IBAPP. 
Pasquali, L. (2003). Psicometria: teoria dos testes na psicologia e na educação. Petrópolis: Vozes.

Pimentel, C. E. (2004). Valores humanos, preferência musical, identificação grupal e comportamento social. Dissertação de mestrado não-publicada, Universidade Federal da Paraíba, João Pessoa.

Putnins, A. (2002). Young offenders, tattoo and recidivism. Psychiatry, Psychology and Law, 9 (1), 62-68.

Santos, W. S. (2008). Explicando comportamentos socialmente desviantes: uma análise do compromisso convencional e afiliação social. Tese de doutorado não-publicada, Universidade Federal da Paraíba, João Pessoa.

Satchithananda, D. K., Walsh, J., \& Schofield, P. M. (2001). Bacterial endocarditis following repeated tattooing. Heart, 85 (1), 11-12.

Schorzman, C. M., Gold, M. A., Downs, J. S., \& Murray, P. M. (2006). Body art: attitudes and practices regarding body piercing among urban undergraduates. Journal of the American Osteopathic Association, 107 (10), 432-438.

Shebani, S. O., Miles, H. F. J., Simmons, P., \& De Giovanni, J. V. (2007). Awareness of the risk of endocarditis associated with tattooing and body piercing among patients with congenital heart disease and pediatric cardiologists in the United Kingdom. Archives of Disease in Childhood, 92 (11), 1013-1014.

Stuppy, D. J., Armstrong, M. L., \& Casals-Ariet, C. (1998). Attitudes of health care providers and students towards tattooed people. Journal Advanced Nursing, 27 (6), 1165-1170.

Tabachnick, B., \& Fidell, L. S. (2006). Using multivariatestatistics ( $5^{\text {th }}$ ed.). New York: Harper Collins.

Vail, A. (1999). Tattoos are like potato chips ... you can't have just one: the process of becoming a tattoo collector. Deviant Behavior, 20 (2), 253-273.

Van de Vijver, F. \& Leung, K. (1997). Methods and data analysisforcross-cultural research. Thousand Oaks, CA: Sage Publications.

Recebido em: 5/3/2009

Versão final reapresentada em: 27/5/2009

Aprovado em: 26/6/2009 